\title{
CHEMICAL CHARACTERIZATION OF SOIL ORGANIC MATTER \\ FRACTIONS BY ANALYTICAL PYROLYSIS-GAS \\ CHROMATOGRAPHY-MASS SPECTROMETRY
}

\section{SAIZ-JIMENEZ *}

Agencia de Medio Ambiente, Junta de Andalucia, Sevilla, and Centro de Edafologia y Biologia Aplicada, C.S.I.C., Sevilla (Spain)

\section{J.W. DE LEEUW}

Delft University of Technology, Department of Chemistry and Chemical Engineering, Organic Geochemistry Unit, De Vries van Heystplantsoen 2, 2628 RZ Delft (The Netherlands)

(Received March 17th, 1985; accepted July 5th, 1985)

\section{SUMMARY}

Curie-point pyrolysis-gas chromatography-mass spectrometry has been used to chemically characterize fulvic acid, humic acid, hymatomelanic acid and polysaccharide fractions of a representative soil. A detailed study of all the pyrolysis products was made. By: comparing the results of this study with previous pyrolysis data for lignins, degraded lignins. polysaccharides, proteins, etc., we have been able to obtain more detailed information aboul the chemical composition of the pyrolysis products from different soil organic matter fractions. It is shown that fulvic acid fractions consist mainly of polysaccharide and/or carbohydrates and polyphenols, that humic acid and humin fractions are complex mixtures of several biopolymers such as polysaccharides, partially degraded lignins, peptides and lipids and that the hymatomelanic acid fraction represents mainly lipid materials.

\section{INTRODUCTION}

Plant residues constitute an important organic component of soils. Living, dying and dead tissues representing wide varieties of chemical substances undergo biochemical and chemical degradation reactions. The compounds produced in this way, the so-called humic substances, are very complex in nature and are thought to be more stable than the starting materials.

The classical method of fractionation of humic substances is based on their solubility in alkalis and acids. The major fractions thus obtained are

\footnotetext{
\# Address for correspondence: Apartado 12.017, 41011 Sevilla, Spain.
} 
humic acid, fulvic acid and humin. A further fractionation of humic acid into hymatomelanic acid, an ethanol-soluble fraction, is sometimes performed. In addition to humic substances, polysaccharides are quantitatively important compounds present in soil organic matter [1].

Numerous investigations have been undertaken to characterize the chemical structure of soil organic matter fractions by means of chemical degradation techniques [2]. More recently, pyrolysis-mass spectrometric studies of different humic fractions and related materials (e.g. fungal melanins, lignins, polysaccharides) have been reported. This analytical approach is used as a fingerprinting technique and clearly shows similarities and differences between different fractions present in soils $[1,3-5]$.

However, to obtain an insight into the structural composition of the organic matter more firm identifications of the pyrolysis products, which reflect structural moieties present within the polymeric matrix, are required.

In this study we used Curie-point pyrolysis-gas chromatography-mass spectrometry (Py-GC-MS) to identify the pyrolysis products of the polysaccharide, fulvic acids, humic acid and humin and also the hymatomelanic acid fraction of a representative soil. By comparing the results of this study with those of previous Py-GC-MS studies of lignins, degraded lignins, polysaccharides and fulvic acids [6,7], we have been able to obtain more detailed information about the chemical composition of the pyrolysis products from different soil organic matter fractions.

\section{EXPERIMENTAL}

\section{Soil sample}

The soil sample used was obtained from the $A_{1}$ horizon of a brown soil (Typic Xerochrept) in the northern part of the province of Huelva, Spain.

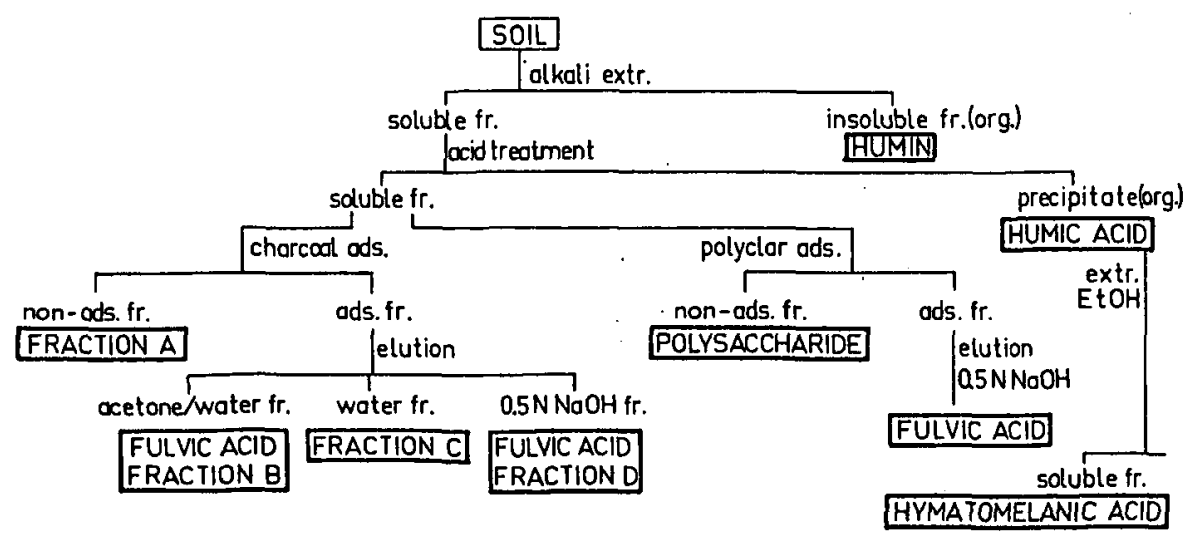

Fig. 1. Fractionation of soil organic matter (for details, see ref. 1). 
TABLE 1

Elemental composition of soil organic matter fractions [1]

\begin{tabular}{llllll}
\hline Fraction & $\mathrm{C}(\%)$ & $\mathrm{H}(\%)$ & $\mathrm{N}(\%)$ & $\mathrm{O}(\%)$ & Ash (\%) \\
\hline Polysaccharide & 37.8 & 6.8 & 2.1 & 53.3 & 22.0 \\
Humin & 55.1 & 6.6 & 4.2 & 34.1 & 2.2 \\
Humic acid & 51.4 & 5.8 & 4.1 & 38.7 & 5.5 \\
Hymatomelanic acid & 57.8 & 7.5 & 1.1 & 33.6 & 2.5 \\
Fulvic acid (Polyclar) & 47.9 & 5.2 & 2.6 & 44.3 & 11.5 \\
Fulvic acid (fraction B) & 49.9 & 6.3 & 1.4 & 42.4 & 6.5 \\
Fulvic acid (fraction D) & 39.5 & 4.3 & 2.5 & 53.7 & 21.1 \\
\hline
\end{tabular}

The altitude was $480 \mathrm{~m}$ and the vegetation consisted of an uncultivated prairie with gramineous plants, Medicago and Trifolium. The sample taken represented a depth of $0-10 \mathrm{~cm}$ and had a $\mathrm{pH}$ of 5.6 in water, a carbon content of $3.5 \%$ and a nitrogen content of $0.4 \%$. The applied fractionation procedure for soil organic matter is described elsewhere [1]. For the reader's convenience we have included Fig. 1, which indicates the different fractions studies and the procedural pathways by which they were obtained. Table 1 shows the elementary composition of the isolated soil organic matter fractions on a dry and ash-free basis [1].

Pyrolysis-gas chromatography-mass spectrometry

The samples were suspended in methanol. One droplet of the suspension (10-20 $\mu \mathrm{g}$ of sample) was applied to a ferromagnetic wire with a Curie temperature of $510^{\circ} \mathrm{C}$. The temperature rise time was about $0.15 \mathrm{~s}$ and the wire was held at the end temperature for $10 \mathrm{~s}$.

The Py-GC-MS analyses were carried out using a pyrolysis unit similar to that described by Meuzelaar et al. [8] modified for use at high temperatures [9]. The pyrolysis products were separated on a capillary glass WCOT column ( $28 \mathrm{~m} \times 0.5 \mathrm{~mm}$ I.D.) coated with CP-Sil 5 ( $1.25 \mu \mathrm{m}$ film thickness) held at $0^{\circ} \mathrm{C}$ for $5 \mathrm{~min}$ and subsequently programmed to $300^{\circ} \mathrm{C}$ at a rate of $5^{\circ} \mathrm{C} / \mathrm{min}$. Helium was used as the carrier gas at a rate of $1.6 \mathrm{ml} / \mathrm{min}$. The chromatograph (Varian Model 3200) was coupled to a Varian-MAT 44 quadrupole mass spectrometer operated in the electron impact (EI) mode at $80 \mathrm{eV}$ and with a cycle time of $2 \mathrm{~s}$.

\section{RESULTS AND DISCUSSION}

Pyrolysis products were identified by comparing their EI mass spectra with mass spectral libraries $[10,11]$ and with mass spectra and $G C$ retention times of standard compounds. Subsequently, Py-GC-MS data obtained 


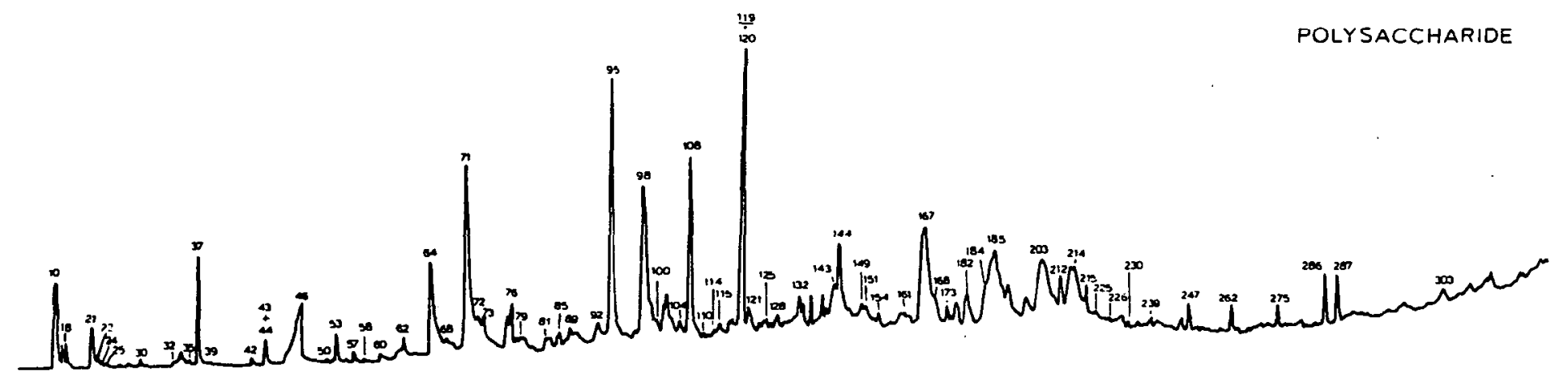

HUMIN

of

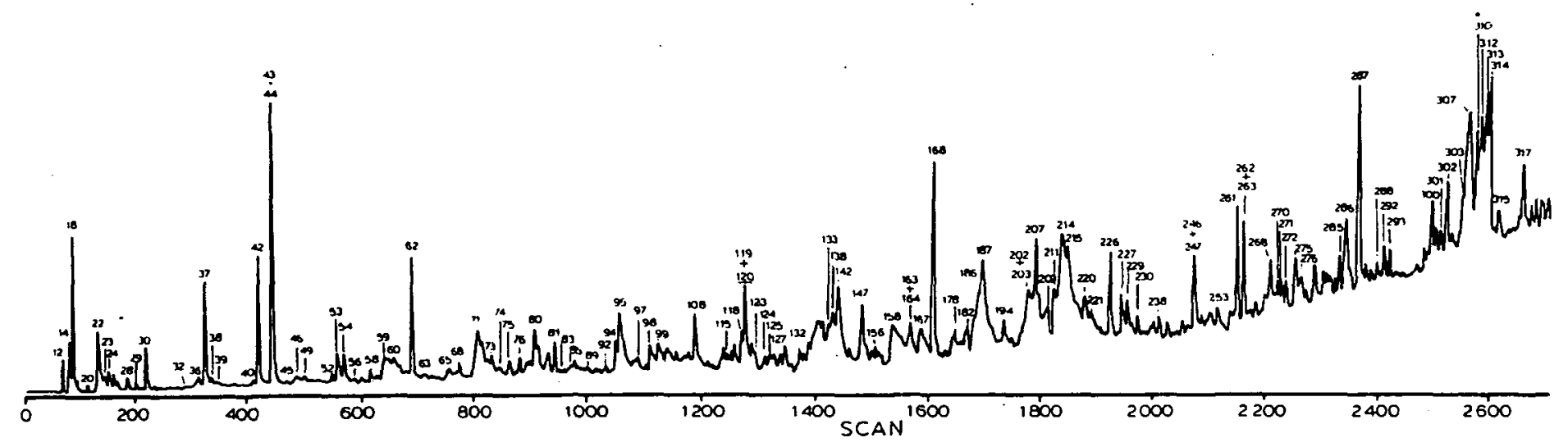

Fig. 2. Pyrolysis-gas chromatography-mass spectrometry of soil polysaccharide and humin. Peak identifications are given in Table 2. Underlined numbers indicate minor contributions to the peak. 
$\underbrace{}_{1}$

HYMATOMELANIC ACID

$\pm$

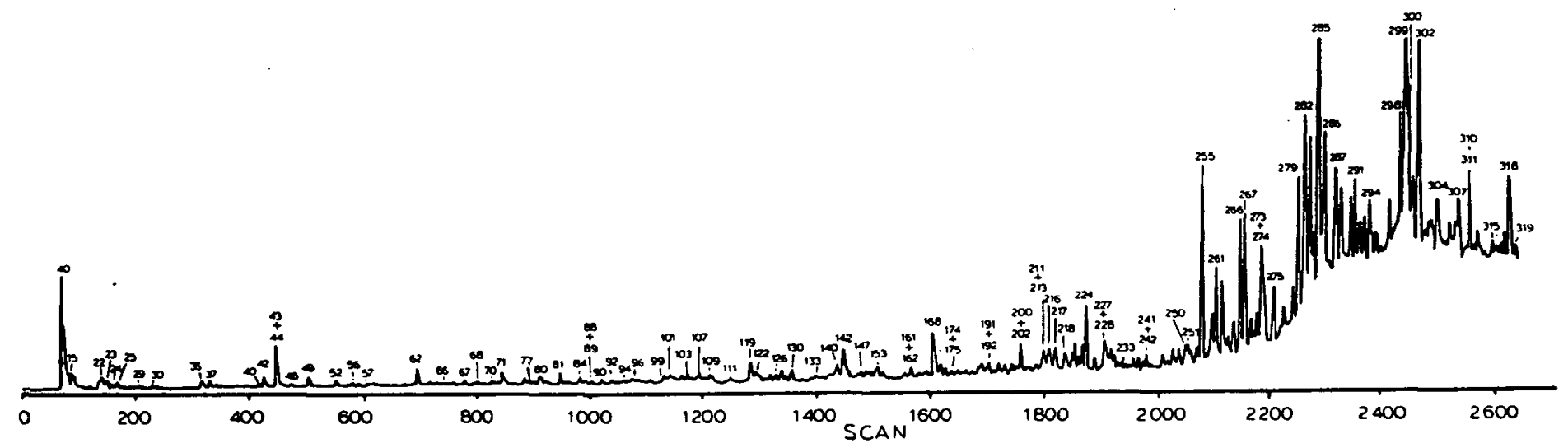

Fig. 3. Pyrolysis-gas chromatography-mass spectrometry of humic acid and hymatomelanic acid. Peak identifications are given in Table 2 . Underlined numbers indicate minor contributions to the peak. 


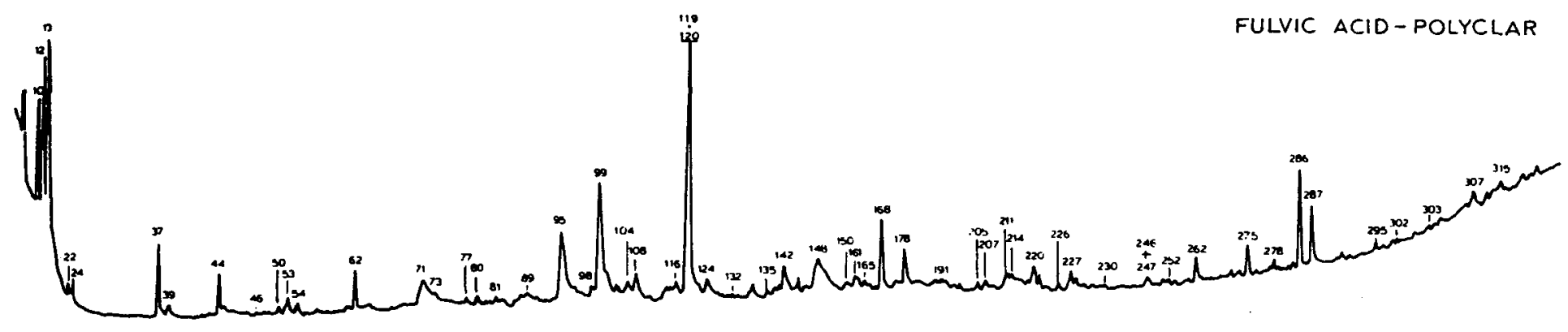

N

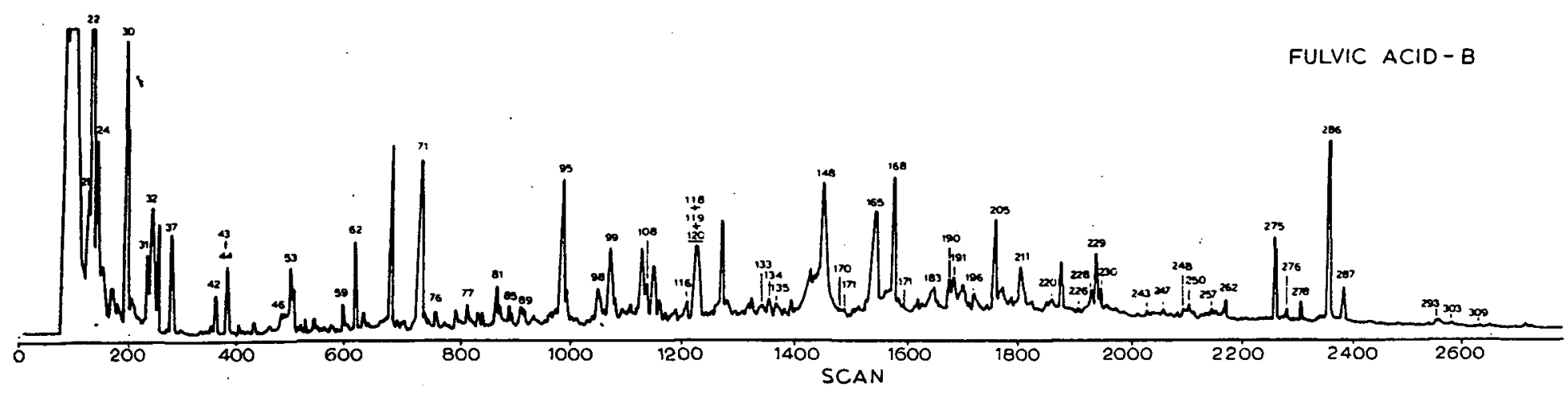




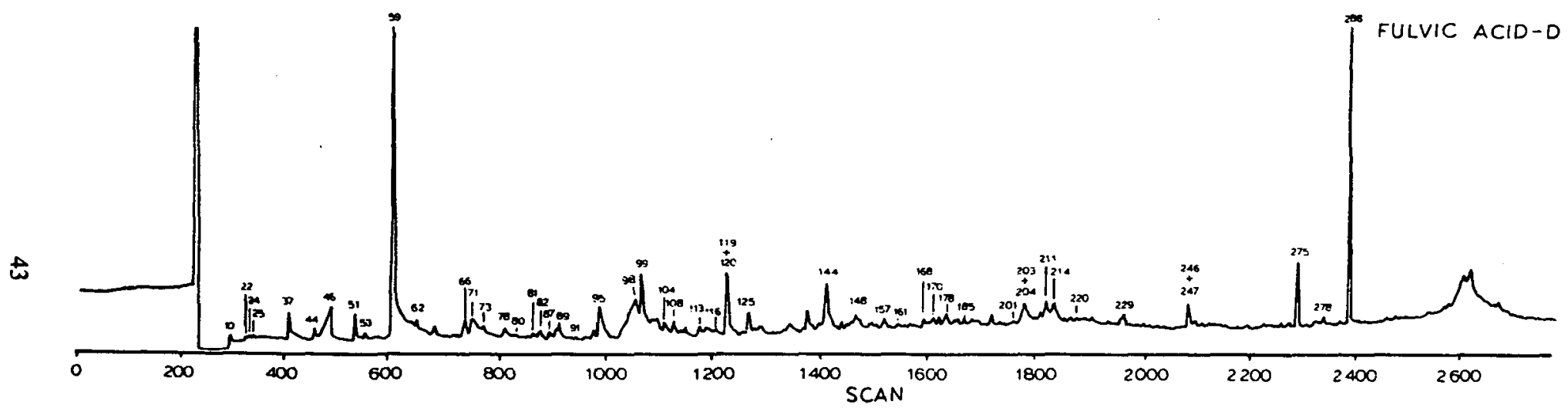

Fig. 4. Pyrolysis-gas chromatography-mass spectrometry of fulvic acid fractions. Peak identifications are given in Table 2. Underlined numbers indicate minor contribution to the peak. 


\section{TABLE 2}

Pyrolysis products of soil organic matter fractions as identified by pyrolysis-gas chromatography-mass spectrometry

\begin{tabular}{|c|c|c|}
\hline Peak No. & Compound & Origin * * \\
\hline 1 & Carbon monoxide & \\
\hline 2 & Carbon dioxide & \\
\hline 3 & Methane & \\
\hline 4 & Ethene & \\
\hline 5 & Ethane & \\
\hline 6 & Hydrogen sulphide & $\operatorname{Pr}$ \\
\hline 7 & Propene & \\
\hline 8 & Propane & \\
\hline 9 & Methanol & Ps, Lg \\
\hline 10 & Sulphur dioxide & \\
\hline 11 & Hydrochloric acid & \\
\hline 12 & Chloromethane & \\
\hline 13 & Acetaldehyde & Ps \\
\hline 14 & 2-Methylprop-1-ene & \\
\hline 15 & But-1-ene & \\
\hline 16 & Buta-1,3-diene & \\
\hline 17 & $n$-Butane & \\
\hline 18 & Methanethiol & $\operatorname{Pr}$ \\
\hline 19 & trans-But-2-ene & \\
\hline 20 & 3-Methylpent-1-ene & \\
\hline 21 & 2-Propenal & Ps \\
\hline 22 & Acetone & Ps \\
\hline 23 & Pent-1-ene & \\
\hline 24 & Furan & Ps \\
\hline 25 & Iodomethane & \\
\hline 26 & $n$-Pentane & \\
\hline 27 & Ethanethiol & $\operatorname{Pr}$ \\
\hline 28 & cis-Pent-2-ene & \\
\hline 29 & Cyclopentadiene & Ps \\
\hline 30 & 2-Methylpropenal & Ps \\
\hline 31 & 3-Buten-2-one & Ps \\
\hline 32 & Butane-2,3-dione & Ps \\
\hline 33 & 2-Methylpentane & \\
\hline 34 & Cyclopentane & \\
\hline 35 & Methyldihydrofuran & Ps \\
\hline 36 & Hex-1-ene & \\
\hline 37 & 2-Methylfuran & Ps \\
\hline 38 & $n$-Hexane & \\
\hline 39 & 3-Methylfuran & Ps \\
\hline 40 & Hexatriene & \\
\hline 41 & Hexadiene & \\
\hline 42 & 3-Methylbutanal & $\operatorname{Pr}$ \\
\hline 43 & 2-Methylbutanal & $\operatorname{Pr}$ \\
\hline 44 & Benzene & Ps, Pr, Lg \\
\hline 45 & Thiophene & \\
\hline 46 & Acetic acid & Ps \\
\hline 47 & 2-Methylcyclobutanone & Ps \\
\hline
\end{tabular}


TABLE 2 (continued)

\begin{tabular}{|c|c|c|}
\hline Peak No. ${ }^{\star}$ & Compound & Origin * \\
\hline 48 & Cyclohexane & \\
\hline 49 & Cyclohexene & \\
\hline 50 & Ethylfuran & Ps \\
\hline 51 & 1,4-Dioxan & \\
\hline 52 & Hept-1-ene & \\
\hline 53 & 2,5-Dimethylfuran & Ps \\
\hline 54 & 2,4-Dimethylfuran & Ps \\
\hline 55 & $n$-Heptane & \\
\hline 56 & Dimethylfuran & Ps \\
\hline 57 & Vinylfuran & Ps \\
\hline 58 & N-Methylpyrrole & $\operatorname{Pr}$ \\
\hline 59 & Pyridine. & $\operatorname{Pr}$ \\
\hline 60 & 5-Methyl-2(5H)-furanone & Ps \\
\hline 61 & Pyrrole & $\operatorname{Pr}$ \\
\hline 62 & Toluene & Pr, Lg \\
\hline 63 & Methylthiophene & \\
\hline 64 & $\gamma$-Crotonolactone & Ps \\
\hline 65 & Dihydropyran & Ps \\
\hline 66 & Oct-1-ene & Lp \\
\hline 67 & Oct-2-ene & Lp \\
\hline 68 & 3-Furaldehyde & Ps \\
\hline 69 & $n$-Octane & Lp \\
\hline 70 & $n-C_{s}$ Fatty acid methyl ester & $\mathrm{Lp}$ \\
\hline 71 & 2-Furaldehyde & Ps \\
\hline 72 & Acetamide & Ps \\
\hline 73 & Benzenemethanol & \\
\hline 74 & Methylpyrrole & $\operatorname{Pr}$ \\
\hline 75 & Methylpyrrole & $\operatorname{Pr}$ \\
\hline 76 & Furfuryl alcohol & Ps \\
\hline 77 & Ethylbenzene & $\mathrm{Pr}, \mathrm{Lg}$ \\
\hline 78 & Methylpyridine & $\operatorname{Pr}$ \\
\hline 79 & 3-Methylcyclopent-2-en-1-one & Ps \\
\hline 80 & $m$ - and/or $p$-xylene & $\mathrm{Pr}, \mathrm{Lg}$ \\
\hline 81 & Styrene & \\
\hline 82 & 2-Methylcyclopent-2-en-1-one & Ps \\
\hline 83 & $o$-Xylene & $\mathbf{L g}$ \\
\hline 84 & Non-1-ene & Lp \\
\hline 85 & $\mathrm{C}_{3}$-Alkylfuran & Ps \\
\hline 86 & Furancarboxylic acid & Ps \\
\hline 87 & $\mathrm{C}_{3}$-Alkylfuran & Ps \\
\hline 88 & $n$-Nonane & $\mathbf{L p}$ \\
\hline 89 & a-Angelicalactone & Ps \\
\hline 90 & $n-C_{6}$ Fatty acid methyl ester & Lp \\
\hline 91 & Dimethylpyridine & $\operatorname{Pr}$ \\
\hline 92 & $\alpha$-Methylbenzenemethanol & \\
\hline 93 & $\mathrm{C}_{4}$-Alkylfuran & Ps \\
\hline 94 & Benzaldehyde & Lg \\
\hline 95 & 5-Methyl-2-furaldehyde & Ps \\
\hline 96 & $\mathrm{C}_{3}$-Alkylbenzene & \\
\hline 97 & $\mathrm{C}_{3}$-Alkylbenzene & \\
\hline
\end{tabular}


TABLE 2 (continued)

\begin{tabular}{|c|c|c|}
\hline Peak No. * & Compound & Origin * \\
\hline 98 & 4-Hydroxy-5,6-dihydro-2H-pyran-2-one & Ps \\
\hline 99 & Phenol & $\mathrm{Ps}, \mathrm{Pr}, \mathrm{Lg}$ \\
\hline 100 & Trimethylcyclopentenone & Ps \\
\hline 101 & $\alpha$-Methylstyrene & \\
\hline 102 & Dec-1-ene & Lp \\
\hline 103 & Iso- $C_{7}$ fatty acid methyl ester & Lp \\
\hline 104 & 2-Hydroxy-3-methyl-2-cyclopenten-1-one & Ps \\
\hline 105 & $\mathrm{C}_{3}$-Alkylpyridine & $\operatorname{Pr}$ \\
\hline 106 & $n$-Decane & Lp \\
\hline 107 & n-C, Fatty acid methyl ester & Lp \\
\hline 108 & 4-Hydroxy-6-methyl-5,6-dihydro-2H-pyran-2-one & Ps \\
\hline 109 & Indene & \\
\hline 110 & Furan-2,5-aldehyde & Ps \\
\hline 111 & o-Cresol & $\mathrm{Lg}$ \\
\hline 112 & $\mathrm{C}_{3}$-Alkylbenzene & \\
\hline 113 & $C_{3}$-Alkylbenzene & \\
\hline 114 & 5-(2-Hydroxyethylidene)-2H-furanone & Ps \\
\hline 115 & Hexane-2,3,4-trione & Ps \\
\hline 116 & 3-Hydroxy-6-methyl-3,4-dihydro-2H-pyran-2-one & Ps \\
\hline 117 & 2-Furyl hydroxymethyl ketone & Ps \\
\hline 118 & p-Cresol & Pr, Lg \\
\hline 119 & Guaiacol & $\mathrm{Lg}$ \\
\hline 120 & Levoglucosenone & Ps \\
\hline 121 & 3-Hydroxy-6-methyl-2H-pyran-2-one & Ps \\
\hline 122 & $n-C_{7}$ Fatty acid & Lp \\
\hline 123 & Ethylstyrene & $\mathrm{Lg}$ \\
\hline 124 & 3-Hydroxy-2-methyl-4H-pyran-4-one & Ps \\
\hline 125 & 3-Acetoxypyridine & Ps \\
\hline 126 & Undec-1-ene & Lp \\
\hline 127 & Benzyl cyanide & $\operatorname{Pr}$ \\
\hline 128 & 5-Hydroxy-2-methyl-4H-pyran-4-one & Ps \\
\hline 129 & $n$-Undecane & Lp \\
\hline 130 & n- $\mathrm{C}_{8}$ Fatty acid methyl ester & Lp \\
\hline 131 & Methylpentane-1,5-dioate & Lp \\
\hline 132 & 2,3-Dihydro-3,5-dihydroxy-6-methyl-4H-pyran-4-one & Ps \\
\hline 133 & Ethylphenol & $\mathrm{Lg}$ \\
\hline 134 & $\mathrm{C}_{4}$-Alkylbenzene & \\
\hline 135 & $\mathrm{C}_{4}$-Alkylbenzene & \\
\hline 136 & $\mathrm{C}_{2}$-Alkylphenol & $\mathrm{Lg}$ \\
\hline $1 \cdot 37$ & $\mathrm{C}_{4}$-Alkylbenzene & \\
\hline 138 & $\mathrm{C}_{8} \mathrm{H}_{9} \mathrm{NO}_{2}$ (chitin) & Ps \\
\hline 139 & Naphthalene & \\
\hline 140 & $n-C_{8}$ Fatty acid & Lp \\
\hline 141 & $\mathrm{C}_{2}$-Älkylphenol & $\mathbf{L g}$ \\
\hline 142 & Methylguaiacol & Lg \\
\hline 143 & 3,5-Dihydroxy-2-methyl-4H-pyran-4-one & Ps \\
\hline 144 & $1,4: 3,6$-Dianhydro- $\alpha$-D-glucopyranose & Ps \\
\hline 145 & Methylnaphthalene & \\
\hline 146 & Dodec-1-ene & $L_{p}$ \\
\hline
\end{tabular}


TABLE 2 (continued)

\begin{tabular}{|c|c|c|}
\hline Peak No. ${ }^{\star}$ & Compound & Origin * \\
\hline$\overline{147}$ & Iso- $C_{9}$ fatty acid methyl ester & Lp \\
\hline 148 & Vinylphenol & $\mathbf{L g}$ \\
\hline 149 & Resorcinol & Ps. Lg \\
\hline 150 & 2-Methylthiophenol & \\
\hline 151 & Anhydrohexose & Ps \\
\hline 152 & $n-C_{9}$ Fatty acid methyl ester & Lp \\
\hline 153 & Methylhexane-1,6-dioate & Lp \\
\hline 154 & 5-(Hydroxymethyl)-2-furaldehyde & Ps \\
\hline 155 & $n$-Dodecane & $\mathbf{L p}$ \\
\hline 156 & Amino acid dimer & Pr \\
\hline 157 & 1-Indanone & \\
\hline 158 & Amino acid dimer & Pr \\
\hline 159 & Methylthiophenol & \\
\hline 160 & $\mathrm{C}_{6}$-Alkylbenzene & \\
\hline 161 & Ethylguaiacol & $\mathbf{L g}$ \\
\hline 162 & $n-C_{9}$ Fatty acid & $\mathbf{L p}$ \\
\hline 163 & Indole & Pr \\
\hline 164 & $\mathrm{C}_{8} \mathrm{H}_{9} \mathrm{NO}_{2}$ (chitin) & Ps \\
\hline 165 & Phthalic anhydride & \\
\hline 166 & Iso- $C_{10}$ fatty acid methyl ester & $\mathbf{L p}$ \\
\hline 167 & 1,4-Dideoxy-D-glycero-hex-1-enopyranos-3-ulose & Ps \\
\hline 168 & Vinylguaiacol & $\mathbf{L}_{\mathbf{g}}$ \\
\hline 169 & Tridec-1-ene & Lp \\
\hline 170 & Indan-1,3-dione & \\
\hline 171 & $1(3 \mathrm{H})$-Isobenzofuranone & \\
\hline 172 & $n$-Tridecane & Lp \\
\hline 173 & $\mathrm{C}_{7} \mathrm{H}_{7} \mathrm{NO}_{3} / \mathrm{C}_{8} \mathrm{H}_{11} \mathrm{NO}_{2}$ (chitin) & Ps \\
\hline 174 & $n-C_{10}$ Fatty acid methyl ester & Lp \\
\hline 175 & Methylheptane-1,7-dioate & $\mathbf{L p}$ \\
\hline 176 & Amino acid dimer & Pr \\
\hline 177 & Amino acid dimer & Pr \\
\hline 178 & 2,6-Dimethoxyphenol & $\mathbf{L g}$ \\
\hline 179 & Amino acid dimer & $\mathbf{P r}$ \\
\hline 180 & Amino acid dimer & Pr \\
\hline 181 & Amino acid dimer & Pr \\
\hline 182 & Eugenol & $\mathbf{L g}$ \\
\hline 183 & Benzenepropionic acid & $\mathbf{L g}$ \\
\hline 184 & $\mathrm{C}_{8} \mathrm{H}_{9} \mathrm{NO}_{2}$ (chitin) & Ps \\
\hline 185 & Levogalactosan & Ps \\
\hline 186 & Amino acid dimer & Pr \\
\hline 187 & Methylindole & Pr \\
\hline 188 & $n-C_{10}$ Fatty acid & $\mathbf{L p}$ \\
\hline 189 & Biphenyl & $\mathbf{L g}$ \\
\hline 190 & Methylphthalic anhydride & \\
\hline 191 & Vanillin & $\mathbf{L g}$ \\
\hline 192 & Iso- $C_{11}$ fatty acid methyl ester & $\mathbf{L p}$ \\
\hline 193 & Amino acid dimer & $\mathbf{P r}$ \\
\hline 194 & cis-Isoeugenol & $\mathbf{L}_{\mathbf{B}}$ \\
\hline 195 & Tetradec-1-ene & $\mathbf{L p}$ \\
\hline
\end{tabular}


TABLE 2 (continued)

\begin{tabular}{|c|c|c|}
\hline Peak No. & Compound & Origin $\star \star$ \\
\hline 196 & Methylphthalic anhydride & \\
\hline 197 & Amino acid dimer & Pr \\
\hline 198 & $n$-Tetradecane & $\mathrm{Lp}$ \\
\hline 199 & $n-C_{11}$ Fatty acid methyl ester & Lp \\
\hline 200 & Methyloctane-1,8-dioate & Lp \\
\hline 201 & 4-Methyl-2,6-dimethoxyphenol & $\mathrm{Lg}$ \\
\hline 202 & Amino acid dimer & Pr \\
\hline 203 & Levoglucosane (mannose?) & Ps \\
\hline 204 & Phthalide & \\
\hline 205 & Dimethyl phthalate & \\
\hline 206 & Amino acid dimer & Ps \\
\hline 207 & trans-Isoeugenol & $\mathrm{Lg}$ \\
\hline 208 & Amino acid dimer & $\operatorname{Pr}$ \\
\hline 209 & Amino acid dimer & $\operatorname{Pr}$ \\
\hline 210 & Ethyl- $\alpha$-ethyl hexanoate & Lp \\
\hline 211 & Acetoguaiacone & $\mathrm{Lg}$ \\
\hline 212 & Trianhydro-2-acetamido-2-deoxyglucose $\left(\mathrm{C}_{8} \mathrm{H}_{9} \mathrm{NO}_{3}\right)$ & Ps \\
\hline 213 & $n-C_{11}$ Fatty acid & Lp \\
\hline 214 & Levoglucosane (glucose) & Ps \\
\hline 215 & $\mathrm{C}_{8} \mathrm{H}_{9} \mathrm{NO}_{3}$ (chitin) & Ps \\
\hline 216 & 3,4-Dimethoxyacetophenone & Lg \\
\hline 217 & Iso- $C_{12}$ fatty acid methyl ester & Lp \\
\hline 218 & Methyl vanillate & $\mathrm{Lg}$ \\
\hline 219 & 4-Ethyl-2,6-dimethoxyphenol & $\mathrm{Lg}$ \\
\hline 220 & (4-Hydroxy-3-methoxy)phenylpropan-2-one & $\mathrm{Lg}$ \\
\hline 221 & $n$-Pentadecane & Lp \\
\hline 222 & Tributyl phosphate & \\
\hline 223 & $n-C_{12}$ Fatty acid methyl ester & $\mathrm{Lp}$ \\
\hline 224 & Methylnonane-1,9-dioate & Lp \\
\hline 225 & $\mathrm{C}_{8} \mathrm{H}_{11} \mathrm{NO}_{4}$ (chitin) & Ps \\
\hline 226 & 4-Vinyl-2,6-dimethoxyphenol & Lg \\
\hline 227 & $n-C_{12}$ Fatty acid & Lp \\
\hline 228 & Propioguaiacone & Lg \\
\hline 229 & Diethyl phthalate & \\
\hline 230 & Vanillic acid & Lg \\
\hline 231 & Dihydroxydimethylbenzaldehyde & Lg \\
\hline 232 & 4-Allyl-2,6-dimethoxyphenol & $\mathrm{Lg}$ \\
\hline 233 & Iso- $C_{13}$ fatty acid methyl ester & Lp \\
\hline 234 & Anteiso- $C_{13}$ fatty acid methyl ester & Lp \\
\hline 235 & Dianhydro-2-acetamido-2-deoxyglucose & Ps \\
\hline 236 & Hexadecadiene & Lp \\
\hline 237 & Hexadec-1-ene & Lp \\
\hline 238 & $n$-Hexadecane & Lp \\
\hline 239 & $\mathrm{C}_{8} \mathrm{H}_{9} \mathrm{NO}_{3}$ (chitin) & Ps \\
\hline 240 & $n-C_{13}$ Fatty acid methyl ester & Lp \\
\hline 241 & Methyldecane-1,10-dioate & Lp \\
\hline 242 & Syringaldehyde & $\mathrm{Lg}$ \\
\hline 243 & Tributyl phosphate & \\
\hline 244 & $n-C_{13}$ Fatty acid & Lp \\
\hline
\end{tabular}


TABLE 2 (continued)

\begin{tabular}{|c|c|c|}
\hline Peak No. & Compound & Origin * \\
\hline 245 & Iso- $\mathrm{C}_{14}$ fatty acid methyl ester & Lp \\
\hline 246 & 4-trans-Propenyl-2,6-dimethoxyphenol & Lg \\
\hline 247 & Biphenol & $\mathbf{L g}$ \\
\hline 248 & $\mathrm{C}_{9}$-Alkylphenol & \\
\hline 249 & Acetosyringone & Lg \\
\hline 250 & $\mathrm{C}_{9}$-Alkylphenol & \\
\hline 251 & Heptadecadiene & $\mathrm{Lp}$ \\
\hline 252 & 4-Propyl-2,6-dimethoxyphenol & $\mathrm{Lg}$ \\
\hline 253 & Heptadec-1-ene & $\mathbf{L p}$ \\
\hline 254 & $n$-Heptadecane & Lp \\
\hline 255 & $n-C_{14}$ Fatty acid methyl ester & Lp \\
\hline 256 & Methylundecane-1,11-dioate & Lp \\
\hline 257 & $\mathrm{C}_{9}$-Alkylphenol & \\
\hline 258 & 4-Propenol-2,6-dimethoxyphenol & Lg \\
\hline 259 & 4-Propanol-2,6-dimethoxyphenol & Lg \\
\hline 260 & $\mathrm{C}_{9}$-Alkylphenol & \\
\hline 261 & Prist-1-ene & Lp \\
\hline 262 & $n-C_{14}$ Fatty acid & Lp \\
\hline 263 & Prist-2-ene & Lp \\
\hline 264 & Anthracene & \\
\hline 265 & 10-Methyl- $C_{14}$ fatty acid methyl ester & Lp \\
\hline 266 & Iso- $\mathrm{C}_{15}$ fatty acid methyl ester & Lp \\
\hline 267 & Anteiso- $C_{15}$ fatty acid methyl ester & Lp \\
\hline 268 & Octadec-1-ene & Lp \\
\hline 269 & Methyl ferulate & $\mathbf{L g}$ \\
\hline 270 & $n$-Octadecane & $L_{p}$ \\
\hline 271 & Iso- $C_{15}$ fatty acid & Lp \\
\hline 272 & Anteiso- $C_{15}$ fatty acid & Lp \\
\hline 273 & $n \cdot C_{15}$ Fatty acid methyl ester & Lp \\
\hline 274 & 2,6-Dimethoxyphenyl-4-propionic acid & $\mathbf{L g}$ \\
\hline 275 & Diisobutyl phthalate & \\
\hline 276 & $n-C_{15}$ Fatty acid & Lp \\
\hline 277 & 10 -Methyl- $C_{15}$ fatty acid methyl ester & Lp \\
\hline 278 & Dialkyl phthalate & \\
\hline 279 & Iso- $C_{16}$ fatty acid methyl ester & $L_{p}$ \\
\hline 280 & Phytadiene & Lp \\
\hline 281 & Nonadec-1-ene & Lp \\
\hline 282 & Iso- $C_{16: 1}$ fatty acid methyl ester & $\mathbf{L p}$ \\
\hline 283 & Anteiso- $C_{16: 1}$ fatty acid methyl ester & Lp \\
\hline 284 & $n$-Nonadecane & Lp \\
\hline 285 & $n-C_{16}$ Fatty acid methyl ester & $L_{p}$ \\
\hline 286 & Dibutyl phthalate & \\
\hline 287 & $n-C_{16}$ Fatty acid & $L_{p}$ \\
\hline 288 & $n-C_{16}$ Fatty acid ethyl ester & Lp \\
\hline 289 & 10-Methyl- $C_{16}$ fatty acid methyl ester & Lp \\
\hline 290 & Iso- $C_{17}$ fatty acid methyl ester & Lp \\
\hline 291 & Anteiso- $C_{17}$ fatty acid methyl ester & Lp \\
\hline 292 & Eicos-1-ene & Lp \\
\hline 293 & n-Eicosane & Lp \\
\hline
\end{tabular}


TABLE 2 (continued)

\begin{tabular}{|c|c|c|}
\hline Peak No. & Compound & Origin * \\
\hline 294 & $n-C_{17}$ Fatty acid methyl ester & $\mathrm{Lp}$ \\
\hline 295 & $n-C_{17}$ Fatty acid & Lp \\
\hline 296 & 10-Methyl- $\mathrm{C}_{17}$ fatty acid methyl ester & Lp \\
\hline 297 & Iso- $C_{18}$ fatty acid methyl ester & Lp \\
\hline 298 & $C_{18: 2}$ Falty acid methyl ester & $\mathrm{Lp}$ \\
\hline 299 & $C_{18 ; 1}$ Fatty acid methyl ester & Lp \\
\hline 300 & $C_{1 k: 1}$ Fatty acid methyl ester & Lp \\
\hline 301 & $n$-Heneicosane & $\mathrm{Lp}$ \\
\hline 302 & $n-C_{18}$ Fatty acid methyl ester & Lp \\
\hline 303 & $n-C_{18}$ Fatty acid & $L_{p}$ \\
\hline 304 & 10-Methyl- $C_{18}$ fatty acid methyl ester & Lp \\
\hline 305 & Iso- $C_{19}$ fatty acid methyl ester & $L_{p}$ \\
\hline 306 & Anteiso- $C_{19}$ fatty acid methyl ester & Lp \\
\hline 307 & Dialkyl phthalate & \\
\hline 308 & Docos-1-ene & $\mathbf{L p}_{\mathrm{p}}$ \\
\hline 309 & $n$-Docosane & Lp \\
\hline 310 & $n-C_{19}$ Fatty acid methyl ester & Lp \\
\hline 311 & Methylhexadecane-1,16-dioate & Lp \\
\hline 312 & Terpenoid & Lp \\
\hline 313 & Terpenoid & Lp \\
\hline 314 & Terpenoid & Lp \\
\hline 315 & $n-C_{19}$ Fatty acid & Lp \\
\hline 316 & Tricos-1-ene & Lp \\
\hline 317 & $n$-Tricosane & Lp \\
\hline 318 & $n-C_{20}$ Fatty acid methyl ester & Lp \\
\hline 319 & $n-C_{20}$ Fatty acid & Lp \\
\hline 320 & Dialkyl phthalate & \\
\hline 321 & $n-C_{21}$ Fatty acid & $\mathrm{Lp}$ \\
\hline 322 & $n-C_{22}^{2}$ Fatty acid & Lp \\
\hline
\end{tabular}

* Peak numbers as shown in Figs. 2-4.

* $\operatorname{Ps}=$ polysaccharide; $\operatorname{Pr}=$ protein; $\mathrm{Lg}=$ lignin; $\mathrm{Lp}=$ lipid.

for well defined polymers such as amylose [12], chitin [13], proteins and peptides [14] and lignins [6] allowed a detailed recognition of typical pyrolysis products.

The reconstructed ion chromatograms of the pyrolysis mixtures obtained from the soil organic matter fractions are shown in Figs. 2-4. The peak numbers in these figures correspond with the numbers mentioned in Table 2. Owing to the vast number of identified compounds in each pyrolysate (e.g., 175 in the hymatomelanic acid fraction), it was not possible to label all peaks in the chromatograms; therefore, only major peaks are indicated in the figures.

Most of the major compounds are well known pyrolysis products of biologically produced substances (polysaccharides, lignins, peptides, lipids, etc.). A number of compounds clearly indicate the presence of pollutants. 


\section{Polysaccharides}

The compounds listed in Table 2 labelled Ps are pyrolysis products which are thought to be characteristic for polysaccharides. These proaucts have been identified in the pyrolysates of cellulose, amylose and soil polysaccharide $[7,12,15]$. The abundant presence of annydrosugars, pyranones and furans in the soil polysaccharide fraction indicates that this fraction consists almost entirely of hardly or non-biodegraded polysaccharides provably originating from residual plant polysaccharides and newly made microbial polysaccharides.

Compounds 185 and 203 have been tentatively identified as "levogalactosan" and "levomannosan". This identification is based on their mass spectral data (identical mass spectra when compared with levoglucosan), on the well known occurrence of galactose and mannose moieties in soil polysaccharides [16] and on the absence of these compounds in the pyrolysates of polyglucoses such as cellulose an amylose.

Compounds 116 (3-hydroxy-6-methyl-3,4-dihydro-2H-pyran-2-one) has been reported previously by Saiz-Jimenez and De Leeuw [7] in the pyrolysate of a soil polysaccharide. This compound is the same as that reported by Van der Kaaden et al. (ref. 12, peak 32) in pyrolysates of amylose.

The distribution pattern of the polysaccharide pyrolysis products encountered in the humin and humic acid fractions is similar to that observed in the soil polysaccharide fraction. In the fulvic acid B fraction the furans and to some extent the pyranones and levoglucosenone are clearly present. However, the anhydrosugars are hardly or not present. This might indicale that in this fraction there are monosaccharide moieties present in structures other than polysaccharides. A phenolic glycoside structure has been proposed for this fraction [17].

In the pyrolysis of carbohydrates, acidic conditions catalyse the formation of furans, in close analogy with the dehydration reactions of carbohydrates under aqueous acidic conditions, while alkaline conditions catalyse the breakdown of the sugar molecule to carbonyl compounds through reverse aldol condensation mechanisms [12]. In the absence of additives (as in this work), both types of reactions take place, but the ratio of furans to carbonyl compounds suggests slightly acidic conditions during pyrolysis. Because all fractions were obtained in the acidic form [1] and suspended in methanol to coat the ferromagnetic wires, it is understandable that the pyrolysis will preferentially produce furans over carbonyl compounds.

Cyclopentenones (compounds 79 and 82) have been reported in pyrolysates of soil organic matter [7,18]. Bracewell et al. [18] considered cyclopentenones as major pyrolysis products of aliphatic polycarboxylic acids and polymaleic acid and suggested that aliphatic polycarboxylic acids are important components in soil organic matter. This suggestion is not supported by previous pyrolysis data [7]. Further, cyclopentenones have been identified 
as pyrolysis products from amylose [12] and are well known burned sugar aroma components [19].

Several pyrolysis products present in the polysaccharide and humin fractions (compounds 72, 125, 138, 164, 173, 184, 212, 215, 225, 235 and 239) have been found in pyrolysates of chitin [13]. It can be speculated that the chitin contribution in soil organic matter is mainly derived from fungi.

\section{Lipids}

It is very likely that a major part of the lipids encountered in the pyrolysates (peaks labelled Lp) are mainly the result of evaporation and are not generated from polymeric frameworks by pyrolysis. The lipid components are predominantly present in the hymatomelanic acid fraction obtained after ethanol extraction of the humic acid fraction. This also indicates that the lipids are mainly freely occurring components, which are easily extractable as such.

Aliphatic hydrocarbons have been detected in almost every plant, animal and microorganism examined, and therefore obvious sources of soil hydrocarbons are plant and animal residues and the soil microbial populations. However, it is improbable that animal residues contribute much directly to soil hydrocarbons. The series of $n$-alkanes and $n$-alkenes ranging from $\mathrm{C}_{2}$ to $\mathrm{C}_{23}$ are encountered in a number of soil organic matter fractions and possibly originate from cuticle materials [20] and microbial populations [21].

Acyclic isoprenoid hydrocarbons such as prist-1-ene, prist-2-ene and phytadiene were identified in the humic acid, hymatomelanic acid and humin fractions. The phytyl side-chain of chlorophyll $a$ is believed to be the source of phytadienes [22]. Recently, it has been reported that tocopherols are likely sources of pristenes, as both flash pyrolysis and thermal degradation of $\alpha$-tocopherol yield prist-1-ene as a major pyrolysis product [23].

Morrison [24] has reported that many substances of a lipid nature, particularly of plant origin, are likely to be present in soils. Such substances would include tocopherols and porphyrins from higher plants and may accumulate as resistant remnants of plant residues undergoing humification. Further, Wagner and Muzorewa [25] considered that lipids extracted from soil organic matter may also be of microbial origin. Microbially synthesized products of a lipid nature in soil may become incorporated into soil humus without undergoing major degradative modifications.

The methyl esters encountered are thought to be procedural artifacts formed from the free fatty acids during the preparation of the pyrolysis samples using methanol as the suspension liquid.

Although the saturated and unsaturated straight-chain fatty acids are the main components in soil organic matter fractions, they are not very characteristic as almost any' organism contains these fatty acids. The relatively abundant presence of iso-, anteiso- and 10-methyl fatty acids with chain 
lengths ranging from $\mathrm{C}_{14}$ to $\mathrm{C}_{20}$ are highly characteristic for a microbial input of fungi and bacteria $[26,27]$. The $\alpha, \omega$-diacids might also be the result of bacterial degradation, although an origin from higher plant waxes cannot be ruled out [28].

There can be little doubt that steroids and terpenoids of various types occur in soils, but in spite of the abundance and variety of terpenoids in plants, there are only a few reports of their presence in soils [29]. Three compounds $(312,313$ and 314$)$ were tentatively identified as terpenoid hydrocarbons, based on their mass spectral fragmentation pattern $(m / z 95$, $109,123,149,163,191,203,207$ ) [30]. These compounds (more detailed structures are as yet unknown) occur only in the humin and hymatomelanic acid fractions.

\section{Lignins}

The compounds listed in Table 2 labelled $\mathrm{Lg}$ are well known and characteristic pyrolysis products of lignins and degraded lignins [6]. The compounds identified show that they are contributions from grasses, higher plants and/or trees, as all three types of lignin building blocks ( $p$-coumaryl, coniferyl and syringyl derivatives) are present.

Obviously, lignin is present in all fractions; however, in the soil polysaccharide and the hymatomelanic acid fractions the lignin contribution is minor. Substantial amounts of lignins are present in the humic acid, the humin and the fulvic acid fractions. The distribution of the lignin pyrolysis products indicates that the lignins are partly biodegraded, as the relative amounts of $\mathrm{C}_{3}$-alkyl components are low and as carbonyl and carboxyl functional groups are clearly present [6].

\section{Proteins and peptides}

A number of pyrolysis products are of a protein origin and are labelled $\mathrm{Pr}$ in Table 2. Among them are the so-called "amino acid dimers", originating from valine, leucine and isoleucine pairs. This type of characteristic pyrolysis product, the structures of which are not yet completely known, are also encountered as major components in the pyrolysates of polyamino acids [14]. The peptides are present mainly in the humin and the humic acid fractions, indicating that the relatively high percentage of nitrogen as measured in these fractions (Table 1) originates from peptides.

\section{Miscellaneous}

A number of components present cannot be ascribed to well defined biopolymers, because they are not known as pyrolysis products of the studied biopolymers or they are not pyrolysis products at all. 
Sulphur dioxide might originate from sulphonated materials (e.g., sulphonated polysaccharides). The sulphur content of different soil polysaccharide fractions ranges between 4 and 10\% [31]. Occasionally, sulphur-containing compounds such as thiophenes and thiophenols have been identified in humic acid and fulvic acid fractions, respectively.

Chlorine compounds (hydrochloric acid and chloromethane) probably arise from the hydrochloric acid employed in the fractionation and purification procedures. At present, no explanation can be given for the presence of iodomethane and 1,4-dioxane.

Among the pyrolysis products some compounds considered as pollutants were identified. Dialkyl phthalates were the most prominent, especially in fulvic acid fractions. The origin of the pollutants may be diverse [32] and they interact with the soil organic matter fractions, either in the soil or during the extraction and fractionation procedures.

\section{Nature of the soil organic matter fractions}

Having discussed the origin of the pyrolysis product, it is now convenient to survey the main groups of compounds identified in each soil organic fraction.

\section{Soil polysaccharide}

The fraction obtained by the Polyclar filtration, called soil polysaccharide, consists almost entirely of polysaccharides. The major pyrolysis products encountered are well known and specific pyrolysis products of polysaccharides. Trace amounts of lignin pyrolysis products and fatty acids are also present.

\section{Humin}

The pyrolysate of this fraction consisted of a complex mixture. The main series of pyrolysis products encountered originate from polysaccharides and lignins. Lipids are significantly present, including alkanes, alkenes, fatty acids, terpenoids and pristenes. The presence of peptides is also evident. Pollutants are minor products.

\section{Humic acid}

The major pyrolysis compounds encountered are lignin derivatives. Polysaccharide products are also clearly present, whilst peptide pyrolysis products are less prominent. Lipids are minor components.

\section{Hymatomelanic acid}

This fraction consists almost entirely of lipids including alkanes, alkenes, acyclic isoprenoid hydrocarbons, fatty acids and aliphatic dicarboxylic acids. Lignin pyrolysis products are minor compounds. 


\section{Fulvic acid (Polyclar)}

The initially retained fraction during the Polyclar filtration, called fulvic acid, contains mainly two series of pyrolysis products. Most abundant are the lignin pyrolysis products. A series of pyrolysis products originating from polysaccharides are also clearly present. Fatty acids and dialkyl phthalates are encountered in minor amounts.

\section{Fulvic acid B (charcoal)}

This fraction shows two main series of pyrolysis products originating from lignins and/or polyphenols and carbohydrates. Dialkyl phthalates are present, in addition to minor amounts of fatty acids.

\section{Fulvic acid D (charcoal)}

Major products in the pyrolysate of this fraction are dialkyl phthalates and pyridine. Lignin and carbohydrate pyrolysis products are less important than in the other fulvic acid fractions.

\section{CONCLUSIONS}

1. In the $A_{1}$ horizon of the studied Typic Xerochrept soil about $10 \%$ of the total organic matter is present as carbohydrate in the soil polysaccharide fraction [1]. In addition, various amounts of polysaccharide moieties are present in the humic fractions.

2. The structures of the pyrolysis products encountered in the humic acid fraction are also observed in more or less pure lignin preparations and in polysaccharides. Hence, there is no need to assume that the pyrolysable part of the humic acid fraction consists of a condensed lignin-polysaccharide structure; a simple mixture of these two biopolymeric substances can explain the results satisfactorily.

3. As already pointed out for the humic acid fraction, the pyrolysis data for the other humic fractions also indicate that there is no direct need to assume that humic substances are generated by condensation reactions of lipids', carbohydrates, amino acids, etc. On the contrary, mixtures of more or less biodegraded biopolymers and originally present low-molecular-weight compounds explain the pyrolysis data very well.

4. The chemical contents of the studied humic fractions are probably determined by the solubility of the individual components (e.g., polyphenolic substances such as lignins dissolve in base, but precipitate on acidification; most polysaccharides remain in aqueous solution; lipids do not dissolve in water but do so in ethanol; lipoproteins, glycolipids, glycoproteins, etc. [33] will end up in almost any fraction).

5. Our results indicate that hymatomelanic acid, a term introduced by Hoppe-Seyler in 1889, cannot be considered as a humic fraction, as it 
consists almost entirely of lipid compounds extractable from the humic acid fraction. Probably extraction of the intact soil with toluene-methanol, for example, prior to fractionation would considerably reduce or completely eliminate the hymatomelanic acid fraction.

6. It is clear from the results obtained from the different fulvic acid fractions that the chemical composition of these fractions is a consequence of the fractionation procedure followed. These data are in agreement with previous observations [34].

7. Finally, the Py-GC-MS technique as applied in this study is a powerful method for chemically characterizing considerable amounts of soil organic matter.

\section{REFERENCES}

1 C. Saiz-Jimenez, K. Haider and H.L.C. Meuzelaar, Geoderma, 22 (1979) 25.

2 M.H.B. Hayes and R.S. Swift, in D.J. Greenland and M.H.B. Hayes (Editors), The Chemistry of Soil Components, Wiley, Chichester, 1978, p. 179.

3 H.L.C. Meuzelaar, K. Haider, B.R. Nagar and J.P. Martin, Geoderma, 17 (1977) 239.

4 K. Haider and H.-R. Schulten, J. Appl. Anal. Pyrol., 8 (1985) 317 and references cited therein.

5 J.M. Bracewell and G.W. Robertson, J. Soil Sci., 27 (1976) 196.

6 C. Saiz-Jimenez and J.W. de Leeuw, Org. Geochem., 6 (1984) 417.

7 C. Saiz-Jimenez and J.W. de Leeuw, Org. Geochem., 6 (1984) 287.

8 H.L.C. Meuzelaar, H.G. Ficke and H.C. den Haring, J. Chromatogr. Sci., 13 (1975) 12.

9 D. van de Meent, S.C. Brown, R.P. Philp and B.R.T. Simoneit, Geochim. Cosmochim. Acta, 44 (1980) 999.

10 S.R. Heller and G.W.A. Milne, EPA/NIH Mass Spectral Data Base, U.S. Department of Commerce, Washington, DC, 1978.

11 Mass Spectrometry Data Center, Eight Peak Index of Mass Spectra, Mass Spectrometry Data Centre, AWRE, Aldermaston, Reading, 1974.

12 A. van der Kaaden, J. Haverkamp, J.J. Boon and J.W. de Leeuw, J. Anal. Appl. Pyrol, 5 (1983) 199.

13 A. van der Kaaden, J.J. Boon, J.W. de Leeuw, F. de Lange, P.J.W. Schuyl, H.-R. Schulten and U. Bahr, Anal. Chem., 56 (1984) 2160.

14 J.J. Boon and J.W. de Leeuw, unpublished results.

15 F. Shafizadeh and Y.L. Fu, Carbohydr. Res., 29 (1973) 113.

16 M.V. Cheshire, J. Soil Sci., 28 (1977) 1.

17 W.G.C. Forsyth, Biochem. J., 41 (1947) 176.

18 J.M. Bracewell, G.W. Robertson and D.I. Welch, J. Anal. Appl. Pyrol., 2 (1980) 239.

19 F.D. Mills and J.E. Hodge, Carbohydr. Res., 51 (1976) 9.

20 M. Nip, E.W. Tegelaar, H. Brinkhuis, J.W. de Leeuw, P.A. Schenck and P.J. Holloway, Naturwissenschaften, in press.

21 J.D. Weete, Phytochemistry, 11 (1972) 1201.

22 D. van de Meent, J.W. de Leeuw and P.A. Schenck, in J.R. Maxwell and A.G. Douglas (Editors), Advances in Organic Geochemistry 1979, Pergamon Press, Oxford, 1980, p. 469.

23 H. Goossens, J.W. de Leeuw, P.A. Schenck and S.C. Brassell, Nature (London), 312 (1984) 440.

24 R.I. Morrison, in G. Eglinton and M.T.J. Murphy (Editors), Organic Geochemistry, Springer-Verlag, Berlin, 1969, p. 558. 
25 G. Wagner and E.I. Muzorewa, in Soil Organic Matter Studies, I.A.E.A., Vienna, 1977, p. 99.

26 A. Ballio and S. Barcellona, Ann. Inst. Pasteur Paris, 114 (1968) 121.

27 J.J. Boon, J.W. de Leeuw, G.J. v/d Hoek and J.H. Vosjan, J. Bacteriol., 129 (1977) 1183.

28 A.S. Kester and J.W. Foster, J. Bacteriol., 85 (1963) 859.

29 F.J. Stevenson, J. Am. Oil Chem. Soc., 43 (1966) 203.

30 M.G. Moshonas and E.D. Lund, Flavour Ind., 1 (1970) 375.

31 C. Saiz-Jimenez and G. Gomez-Alarcon, unpublished results.

32 D.B. Peakall, Residue Rev., 54 (1975) 1.

33 J.S. D'Arrigo, C. Saiz-Jimenez and N.S. Reimer, J. Colloid Interface Sci., 100 (1984) 96.

34 B.G. Murzakov, Symp. Biol. Hung., 11 (1972) 179. 